$$
\text { Jean-Jacques Wunenburger }
$$

\title{
Introduction. Structures et fonctions des imaginaires de l'altérité (alter-hétéros)
}

\section{STRUCTURES AND FUNCTIONS OF OTHERNESS IMAGINARIES (ALTER-HÉTÉROS)}

Abstract: This paper approaches some fundamental philosophical problems, which are more or less present in the contributions. Otherness is not only a specific category of the collective imaginary, but also its "fuel". How could imagination make the difference between what is real and what is imagined? How could this difference produce such a large range of varieties that can reach the extreme of alienation from oneself or from the others? How can the varieties of the imagination give birth to the topics of alteration, altercation, alternation? And how do these relationships create a specific domain in which ethics and aesthetics intermingle?

Keywords: Identity; Difference; Alteration; Altercation: Alternation; Alienation; Exclusion; Fusion, Creativity; Phobia.

\section{JEAN-JACQUES WUNENBURGER}

Université Jean Moulin, Lyon, France jean-jacques.wunenburger@wanadoo.fr

DOI: $10.24193 /$ cechinox.2019.36.01
Tos imaginaires portent sur le monde 1 et les hommes, et dans les deux cas, nous pouvons soit les représenter comme semblables voire comme identiques à nous (ce qui risque d'être déjà une fiction si chaque être est absolument singulier), soit comme dissemblables, hétérogènes, radicalement autres. La langue française, qui dérive aussi bien du grec ancien que du latin, puise généralement dans les deux dictionnaires pour désigner cette charge : Identité : autos, idio, homois, tautotêta, en grec, idem en latin; Hétéro, Allos, hétérotêta, xenos, en grec, alter, en latin, etc. Comment approcher ces lignes flottantes de partage du multiple, quels obstacles cognitifs s'y cachent et comment cette matrice intellectuelle vient-elle innerver les scénarios de l'imaginaire?

\section{L'altérité comme essence de l'imaginaire}

U ne des plus anciennes préoccupations philosophiques consiste à valoriser la ressemblance (entre la copie et le modèle par exemple), même la ressemblance la plus parfaite, l'homothétique, 
l'isomorphique plutôt que la différence. Platon subordonne le questionnement philosophique à la capacité de distinguer une image homéomorphe, qui est à la ressemblance de son modèle tout en n'étant pas son double, sinon il n'y aurait plus d'image, et une image leurre, qui imite fallacieusement la ressemblance mais en étant réellement autre (le galop du cheval du sculpteur donne l'impression d'être vrai alors que ses mouvements sont erronés anatomiquement). La vraie et la fausse ressemblance (illusion) donnent lieu d'ailleurs à des différences d'appellation : Platon nomme eikon l'image vraiment ressemblante, eidolon l'image faussement ressemblante ${ }^{1}$.

Quant à la différence, elle peut être pensée seulement comme une variation interne, un petit écart à l'intérieur d'une ressemblance globale, essentielle (le changement de couleur d'une même forme), mais la plupart du temps elle se donne à penser comme sans rapport, sans relation, sans intersection, sans commune mesure avec l'entité de départ : elle est ainsi vraiment l'autre, le tout autre. Dans le premier cas, la représentation sensible ou intellectuelle fait prévaloir le même sur l'autre et s'engage dans une démarche de rassemblement, de connaturalité voire de fusion ; dans le second cas, elle se heurte à une hétérogénéité radicale, qui doit faire appel à d'autres attributs descriptifs, étrangers au premier, ouvrant ainsi un espace d'extériorité, d'étrangeté, d'exclusion. Conceptuellement le même et l'autre appartiennent bien à deux registres cognitifs que l'on ne peut ni confondre ni rapprocher.

À première vue, il existe bien aussi deux types d'imagination : l'une qui reproduit le même, à partir du perçu ou du pensé, mais en le faisant varier, ou en l'amplifiant (rêverie bachelardienne), l'autre qui imagine autre chose que ce qui est vu et connu ici et maintenant. Pourtant les deux partagent une même fonction, car toute imagination est indiscutablement une altération de ce qui est donné initialement, ce qui fait qu'il n'existe que des imaginaires de l'altération et de l'altérité, même lorsqu'ils se portent sur quelque chose d'identique! Comme toute image doit être autre que son modèle, sous peine de ne plus être distinguée de lui (sosie, reproduction à l'identique, fac-similé), toute production d'image mentale ou matérialisée, tout récit d'une histoire, intègrent une part de dissemblance et d'altérité. Donc l'altérité n'est pas un type, une catégorie particulière d'imaginaire mais qualifie l'imagination elle-même. Donc le titre du colloque confine bien en un sens au pléonasme.

En d'autres manières de parler, toute perception rencontre ce qui est présent, en allemand heideggérien, «da-sein ». L'imagination, définie comme représentation " in absentia ", cherche au contraire, à se représenter ce qui n'est pas là, mais a pu exister, peut exister, ou existe ailleurs. Imaginer c'est se représenter autre chose que ce qui est sous les yeux. Donc toute imagination implique une altérité, qui rend possible l'altération. Finalement toute imagination des personnes et des choses, des humains ou du monde, est une mise en œuvre d'une altérité, l'imagination étant la faculté même du changement, de la variation, de la transformation du réel, et donc de l'irréel ${ }^{2}$. Mais paradoxalement, peut-on donner un contenu à l'altérité, à l'altération qui ne soit pas en partie prélèvement ou projection de ces identités préalables ? C'est bien pourquoi les représentations de l'altérité parviennent mal à restituer cette extériorité 
résistante sans réutiliser des traits de la réalité première. Rien de plus parlant en ce sens que les imaginaires de l'animalité, type d'une altérité non humaine, qui ne sont souvent que des projections de l'humain (anthropomorphisme).

\section{Les transgressions de la différence}

P ourtant malgré cette difficulté à nommer, imager, raconter l'altérité en d'autres termes que ceux déjà connus, l'imagination s'emballe souvent en cherchant à accentuer l'image de l'altérité par des métaphores, symboles et surtout des affects intenses, positifs (attirance du mystérieux, du sublime, etc.) ou négatifs, de crainte, peur, répulsion, haine (le mal amplifié en image diabolique ou satanique). La question se révèle donc plus difficile : une fois posé un autre sujet que moi, différent donc, existe-t-il une capacité de l'imagination à le représenter comme autre, différent, mais en respectant son identité mêlée, complexe, hybride (faite de même et d'autre) ? Ou l'imagination engendre-t-elle toujours une représentation déformée et déformante, qui accentue ou diminue la différence ? Cette hyper-bolisation ou hypo-bolisation de l'autre ouvre alors la porte soit à des rejets de l'altérité, inassimilable, incompatible, répulsive soit à l'inverse à des attractions qui annihilent la différence, entrainant des affects et pulsions fusionnels (le « tout autre " divin avec lequel fusionne le mystique). C'est bien pourquoi Aristote soutenait déjà que les récits (au théâtre comme mythos) peuvent montrer l'homme tel qu'il est, pire qu'il n'est ou meilleur qu'il est ${ }^{3}$.

La question est donc bien de comprendre comment l'imagination qui est toujours altération générique, peut osciller entre une altérité réversible, centripète, attirée vers une matrice identitaire, qui inspire des affects de sécurité, de fusion, de régression, et une altérite irréversible, surdéterminée, radicalisée, qui s'accompagne d'affects négatifs, centrifuges, pouvant conduire à l'exclusion ou l'élimination de cette altérité.

Le programme de réflexion consiste donc bien à affronter trois points de vue :

- quel est cet autre quengendrent l'image, le fantasme, l'imagination, l'imaginaire à travers la rêverie, la mythification? Comment peut-on typifier, classer et subdiviser les statuts et les attributs de cette altérité imaginaire ? Les catégories littéraires ou cinématographiques du merveilleux, du fabuleux, de la fantasy, du fantastique, de l'utopique, etc. illustrent bien ces variations du statut de l'altéritét. Dans tous les cas quel est le volant de manœuvre de cet écart que remplit l'imagination ? Comment se construisent ces différences par rapport au modèle, connu ou inconnu ? Quel est le pouvoir de l'imagination à se donner un contenu nouveau ?

- comment l'altérité effective, factuelle, prise en charge par l'imaginaire peut-elle être dramatisée, amplifiée, dualisée par les représentations et les affects positifs mais surtout généralement négatifs (xénophobie, racisme)?

- comment à l'inverse la différence peut-elle être gommée, absorbée, niée, par projection identitaire?

Si la question de l'hétérophobie, par ses effets spectaculaires, semble la forme la plus visible et sensible du régime d'altération de l'imaginaire, il importe néanmoins de ressaisir l'ambivalence et la bipolarité de l'imaginaire, et donc de prendre en 
compte le tropisme contraire, c'est-à-dire une érotique participative, phagocytante, cannibalisante, qui vient déconstruire, faire imploser l'identité autre de l'autre. Bref l'imaginaire a bien à voir avec un jeu subtil de même et d'autre, exposé au double risque d'une hypertrophie de l'autre, qui le rejette dans un ailleurs, ou d'une hypotrophie qui ne parvient pas à le restituer comme différent. Ainsi retrouvons-nous au cœur de l'imaginaire l'ambivalence des passions opposées, haine et amour, poussées à leur intensité maximale, définissant alors une pathologie.

Dans ce cas, il convient non seulement de ne pas enfermer le problème dans un cas de figure unilatéral, mais de penser les oscillations entre le trop et le trop peu de la différence, tantôt comme des formes de richesse, tantôt comme des formes d'appauvrissement et de dérèglements. Si tout nest pas réductible à un juste milieu, à une composition mixte équilibrée, il s'agit d'accompagner les processus qui rajoutent ou retranchent des traits, pour coïncider avec une créativité de l'imagination (L'amour chez Stendhal est " cristallisation ", c'està-dire une projection de valeurs idéalisées). Mais en s'exposant aux extrêmes, l'imagination perd souvent les potentialités de son imaginaire en accédant à des représentations figées, stéréotypées, réifiées, stérilisantes. Il reste qu'il n'est sans doute pas aisé de définir la ligne de crête à partir de laquelle les excès et les défauts imaginés deviennent risqués, dangereux, destructeurs et donc aliénants pour le sujet imaginant.

L'altérité permet donc de se confronter à un double problème classique de l'imagination : jusqu'où l'imagination peutelle faire varier le réel pour développer sa créativité (passage d'une biographie à une histoire romanesque, par exemple), et quelle est la ligne de séparation entre un imaginaire de libre variation créative et un autre qui expose l'imaginaire sans limite à aliéner le sujet ? Quand l'imaginaire de l'altérité devient-il aliénant pour le sujet imaginant ?

\section{Les usages de l'altérité}

$\mathrm{P}$ our préparer le terrain à ces questionnements fondamentaux, il convient d'aborder plusieurs dimensions du problème :

\section{Génération de l'hétérotypie identitaire}

L'expérience spontanée de l'« alter ego » nous confronte à une représentation de la différence, qui si elle nétait pas perçue, empêcherait la constitution de notre propre identité. Nous ne pouvons, en effet, nous représenter nous-mêmes qu'en nous dédoublant par la réflexion comme en un miroir, qui fait surgir une identité déjà multiple. La thématique du miroir comme aide à la conscience de soi, a bien comme enjeu de nous exposer à une image qui est à la fois mienne et autre ${ }^{5}$. Cette altérité primaire consubstantielle à la donation du Moi peut se doubler à son tour d'une altérité secondaire, qui consiste en un imaginaire narratif du passé, du présent et du futur qui vient diffracter voire éparpiller le Moi. À la tension du même et de l'autre de la spécularité initiale s'ajoute, comme l'a souligné P. Ricoeur, une tension entre un «idem » et un "ipse ", qui sont séparés et reliés par une autofiction, cet imaginaire de représentation (la mimesis comme mise en scène) de notre identité Par ces emboitements qui s'interpénètrent au cours de la vie, l'identité apparaît donc 
comme traversée de différenciation, altération, voire opposition, dessinant ainsi au moins une figure plurielle et complexe de soi. Herméneutique comme psychanalyse ont montré comment l'imagination nous aide à faire émerger une identité, qui loin dêtre monolithique, unidimensionnelle, vit de ses polarités et tensions.

\section{Variations sémantiques et polarisa-} tions des images de l'altérité

Comment rendre compte des générations imaginaires de l'altérité au-delà du Moi ? Comment s'opèrent en général les productions imaginaires lorsquelles minimisent ou maximisent les différences entre le Moi et le Non-moi ? Ces opérations iconiques et narratives varient-elles ellesmêmes selon les domaines d'application ? Comment l'altérité est-elle conjuguée dans des champs différents ? Peut-on dégager une imago-logique, unique ou non, de l'hétérotopie ou de l'hétérochronie ? Les imaginaires de l'espace - choses et personnes séparées de moi - obéissent-ils aux mêmes dispositifs fantastiques que les imaginaires temporels, de ce qui est passé et de ce qui est futur ? et comment se rencontrent-ils dans des totalités configurées?

Il convient de sérier d'abord les schèmes des variations. Au premier niveau, minimaliste, la différence commence par prendre les formes du double, de la gémellité, avant de générer de petites différences de degré qui ne se confondent pas avec celles de nature. Lorsque la différence saute le pas vers une substance propre, elle peut relever du contraste, de l'opposition, du contraire ou du contradictoire. Il existe donc bien une échelle de variation et de mesure de l'écart qui reproduit les catégories de différenciation de la perception sensible et de l'intellection logique.

S'agissant ensuite des modalités opératives de l'altérité imaginée, on peut dégager au moins quatre fonctions performatives :

- celle de l'altération esthétique : l'imagination procède alors à des variations de profils, au sens de Husserl (Abschattun$\mathrm{gen})^{7}$, comme dans la peinture où la représentation épurée, stylisée, fait surgir une apparence différente de son modèle (la nature morte copie et transfigure l'apparence des choses) ${ }^{8}$;

- celle de l'aliénation ontologique au sens où Hegel distingue la sortie de soi du devenir étranger à soi (entaüsserung, entfremdung $)^{9}$. Tel serait l'imaginaire qui fait surgir des êtres monstrueux ou merveilleux totalement inexistants dans le réel ;

- celle de l'altercation performative où l'imagination met en scène un réel à venir, à réaliser, à accomplir (visions utopiques, messianiques, millénaristes);

- celle de l'alternance, sur le modèle du renversement en son contraire, de l'énantiodromie ${ }^{10}$, où le réel est remplacé par une image contraire (image du " monde inversé »).

Sans être exhaustif, on peut reconnaitre quelques procédures de gestation d'un imaginaire différent, mais qui modifie plus moins, selon des intentions et résultats propres, le réel. Cette cartographie sert sans doute de support aux imaginaires de l'altérité en les associant à des règles de production spécifiques, qui empêchent de confondre entre eux tous les mondes « autres ».

\section{Enjeux éthiques des hétérotopies}

Outre cette question de génération des imaginaires de l'altérité, il s'agit encore 
de faire varier les réponses selon les champs d'application : personnes, choses, ailleurs géographiques, altérités temporelles, passé et futur. Leurs portées ne s'équivalent sans doute pas. Il existe des imaginaires de pure portée esthétique, d'autres de portée praxéologique, qui prennent des valeurs propres dans des registres spécifiques. Ainsi les nouveaux immigrants aux États-Unis après la découverte de l'Amérique pouvaient se représenter la nature vierge sur le modèle d'une terre promise, d'un paradis retrouvé, d'un imaginaire cosmologique idéalisé, tout en se représentant les habitants indiens comme des humains dégénérés sauvages, en les excluant donc d'une nature humaine commune et partagée. Il en résulte que les déformations imaginaires de l'espace et du temps, des hommes et des mondes, tout en étant ouvertes à des polarités négatives ou positives, n'ont pas toujours les mêmes effets. Si la nature paradisiaque servait de mobile d'exaltation à la possession des terres, l'imaginaire du bon sauvage conduisait à un ethnocide afin de faire triompher les enfants du "bon » Dieu $^{11}$.

Cette dimension de l'imaginaire de l'altérité anthropologique ouvre donc sur la question éthique : quel est l'usage de cet imaginaire, bon ou mauvais, comment en faire un adjuvant cognitif ou un parasite pathogène ? Comment éros et éris (amour et haine) viennent guider l'imagination ? Enfin quels rôles jouent ces imaginaires de l'autre ? Appauvrissent-ils, déforment-ils l'autre réel, ou participent-ils d'une pré connaissance, d'une préfiguration, d'une schématisation transcendantale ? Autrement dit, quel est le degré de vérité, de cohérence, de croyance aussi de ces imaginaires?
L'imagination une fois libérée de la nécessité du réel, peut donner libre cours à ses fantaisies et explorer tous les mondes possibles. C'est bien pourquoi les imaginaires peuvent mettre en scène des altérations des paysages, des usages, des normes du monde réel (la dramaturgie des enfers et du Jugement dernier, la sexualité sans tabous d'un Sade, etc.). Mais dès lors que cet imaginaire n'est plus un simple jeu de représentations mais inspire et imprègne des actions, les visages de l'altérité sont porteurs de transgressions et conflits qui touchent aux valeurs culturelles et morales. Baisser la barrière de l'altérité en laissant le désir identitaire et fusionnel s'y alimenter peut conduire par exemple à l'inceste (l'altérité de la mère étant transgressée pour devenir un fantasme de possession et de fusion). Intensifier l'étrangeté de l'altérité est pousser à la haine et au meurtre de cet autre hyperbolique. Faut-il dès lors domestiquer, censurer, sanctionner des imaginaires dès lors que leur débordement sur le réel entraine une négation du réel ? Faut-il traiter, soigner des imaginaires sans limites (chez le psychopathe) ? Ou bien ces imaginaires déformés et déformants peuvent-ils être sublimés, transformés en symboles et mythes, pour faire passer les fantasmes du stade primaire de passage à l'acte au stade secondaire (comme dans le travail du rêve chez Freud) qui est œuvre de langage et non de praxis?

En somme les imaginaires de l'altérité désignent un champ d'investigations complexe qui doit d'abord prendre la mesure que l'imagination est « sui generis » une production et une exploration d'autre chose, qui se différencie du réel ou du rationnel. Une fois défini le territoire de l'altérité, il apparaît que l'imagination peut inventer des arborescences d'altérité, les unes déficitaires, et donc 
euphémisées dans leur écart et leur pluralisation; les autres surdéterminées, accentuant le non identique, le non ressemblant, le non commun, jusqu'à rendre irreprésentable la négativité ; enfin une altérité mixée, hybride, faite de ce jeu de même et d'autre. Nul doute que ces trois polarisations disposent d'un pouvoir de fascination, de déréalisation qui contribuent à des vécus pathologiques et esthétiques intenses. Il reste cependant toujours le risque qu'aux deux extrêmes, l'imaginaire induise des comportements régressifs, dangereux voire inhumains, ouvrant la porte aux pathologies transgressives voire criminogènes. Au-delà des manifestations et opérations de l'imagination, il importe donc moins de brider l'imagination que d'en limiter les effets ravageurs par un travail de symbolisation, métaphorisation, mythisation qui lui confère le statut d'un monde autre, mais protégé par une visée propre qui ne le rende jamais coextensif à la réalité. L'imaginaire de l'altérité est une voie royale pour briser, doubler, élargir le monde réel, au-delà de ses limites contraintes, mais en lui attribuant, par les rêveries et l'art, une fonction d'ouverture, de production de sens et non de confusion avec le réel.

\section{Bibliographie}

Aristote, La poétique, Paris, Seuil, 1980.

Bachelard, Gaston, L'air et les songes, Paris, Corti, 1990.

Bourgeois, B., Le vocabulaire de Hegel, Paris, Ellipses, 2011.

Bureau, L., J. Ferrari, J.J. Wunenburger (éd.), La rencontre des imaginaires entre Europe et Amériques, Paris, L'Harmattan, 1993.

Chelebourg, Christian, L'imaginaire littéraire, Paris, A. Colin, 2005.

Lacan, Jacques, « Le stade du miroir », dans Écrits I, réed. 1999. Voir notre développement dans La vie des images, 2eme éd., Grenoble, PUG, 2002.

Dufourcq, Annabelle, La dimension imaginaire du réel dans la philosophie de Husserl, New York, Springer, 2010.

Hegel, Esthétique, rééd., Paris, Garnier-Flammarion, 2009.

Platon, Le Sophiste, Paris, Les Belles Lettres. 2008.

Ricoeur, Paul, Soi-même comme un autre, rééd. Points essais, 2014

Ricoeur, Paul, Temps et récit, I à III, Paris, Seuil, 1991.

Wunenburger, Jean-Jacques, Philosophie des images, 2ème éd. Paris, PUF, 2001.

\section{Notes}

1. Voir Platon, Le Sophiste, Les belles-lettres; et nos développements dans Philosophie des images, 2ème éd. Paris, PUF, 2001.

2. Pour G. Bachelard l'imagination est la faculté non de produire des images mais de les transformer. «On veut toujours que l'imagination soit la faculté de former des images. Or elle est plutôt la faculté de déformer les images fournies par la perception, elle est surtout la faculté de nous libérer des images premières, de changer les images... Le vocable fondamental qui correspond à l'imagination, ce n'est pas image, c'est imaginaire. Grâce à l'imaginaire, l'imagination est essentiellement ouverte, évasive. Elle est dans le psychisme humain l'expérience même de l'ouverture, l'expérience même de la nouveauté. [...]. » L'air et les songes, Paris, Corti, 1990, p. 7-8.

3. Aristote, La poétique, Paris, Seuil, 1980. 
4. Voir Christian Chelebourg, L'imaginaire littéraire, Paris, A. Colin, 2005.

5. Voir J. Lacan, «Le stade du miroir », dans Écrits I, réed. 1999. Voir notre développement dans La vie des images, 2eme éd., Grenoble, PUG, 2002.

6. Voir P. Ricoeur, Soi-même comme un autre, rééd. Points essais, 2014 ; Temps et récit, I à III, Paris, Seuil,1991.

7. Voir Annabelle Dufourcq, La dimension imaginaire du réel dans la philosophie de Husserl, New York, Springer, 2010.

8. Voir Hegel, Esthétique, rééd., Paris, Garnier-Flammarion, 2009.

9. Voir B. Bourgeois, Le vocabulaire de Hegel, Paris, Ellipses, 2011.

10. Thème qui apparaît chez Héraclite, Platon, à la Renaissance et plus récemment chez C.G. Jung.

11. Voir L. Bureau, J. Ferrari, J.J. Wunenburger (éd.), La rencontre des imaginaires entre Europe et Amériques, Paris, L'Harmattan, 1993. 